\title{
Improved RIP Conditions for Compressed Sensing with Coherent Tight Frames
}

\author{
Yao Wang ${ }^{1,2}$ and Jianjun Wang ${ }^{3}$ \\ ${ }^{1}$ School of Mathematics and Statistics, Xian Jiaotong University, Xi'an 710049, China \\ ${ }^{2}$ Shenyang Institute of Automation, Chinese Academy of Science, Shenyang 10016, China \\ ${ }^{3}$ School of Mathematics and Statistics, Southwest University, Chongqing 400715, China \\ Correspondence should be addressed to Jianjun Wang; wjj@swu.edu.cn
}

Received 1 February 2017; Accepted 12 April 2017; Published 15 May 2017

Academic Editor: Daniele Fournier-Prunaret

Copyright (c) 2017 Yao Wang and Jianjun Wang. This is an open access article distributed under the Creative Commons Attribution License, which permits unrestricted use, distribution, and reproduction in any medium, provided the original work is properly cited.

This paper establishes new sufficient conditions on the restricted isometry property (RIP) for compressed sensing with coherent tight frames. One of our main results shows that the RIP (adapted to $D$ ) condition $\delta_{k}+\theta_{k, k}<1$ guarantees the stable recovery of all signals that are nearly $k$-sparse in terms of a coherent tight frame $D$ via the $l_{1}$-analysis method, which improves the existing ones in the literature.

\section{Introduction}

Compressed sensing (CS) has received much recent attention in many fields, for example, information science, electrical engineering, and statistics [1-10]. A key problem in CS is to recover a nearly sparse signal from considerably fewer linear measurements. Typically, it takes the following model:

$$
\mathbf{y}=\Phi \mathbf{f}+\mathbf{z}
$$

where $\mathbf{y} \in \mathbb{R}^{m}$ is a vector of observed measurements, $\mathbf{f} \epsilon$ $\mathbb{R}^{n}$ is an unknown signal needed to be estimated, $\Phi \in$ $\mathbb{R}^{m \times n}$ is a given measurement matrix, and $\mathbf{z} \in \mathbb{R}^{m}$ is a vector of measurement errors. Considering the fact that $\mathbf{f}$ is sparse or nearly sparse in terms of an orthogonal basis, one straightforward approach is to find the sparsest solution of (1) by $l_{0}$ minimization. However, it is well known that solving an $l_{0}$ minimization problem directly is NP-hard in general and thus is computationally infeasible for even moderate size setting [11].

To efficiently estimate $\mathbf{f}$ in the high-dimensional setting, the most popular strategy is to replace the $l_{0}$ norm with its closest convex surrogate, the $l_{1}$ norm, which leads to the following $l_{1}$ norm minimization:

$$
\begin{aligned}
\min _{\mathbf{f} \in \mathbb{R}^{n}} & \|\mathbf{f}\|_{1} \\
\text { subject to } & \|\Phi \mathbf{f}-\mathbf{y}\|_{2} \in B,
\end{aligned}
$$

where $\|\mathbf{f}\|_{1}=\sum_{i=1}^{n}\left|\mathbf{f}_{i}\right|$ and $B$ is a bounded set determined by the noise structures. It is obvious that (2) is a convex optimization problem and thus can be solved efficiently in polynomial time. Therefore, the $l_{1}$ minimization method (2) has been widely used in compressed sensing and other related problems.

There are a number of practical applications in signal and image processing point to problems where signals are not sparse in terms of an orthogonal basis but in terms of an overcomplete and tight frame (see [12-16], and the references therein). In such contexts, the signal $\mathbf{f}$ can be expressed as $\mathbf{f}=D \mathbf{x}$, where $\mathbf{x} \in \mathbb{R}^{d}$ is a sparse or nearly sparse vector. One natural way to recover $f$ is first solving the $l_{1}$ minimization problem (2) with the decoding matrix $\Phi D$ instead of $\Phi$ to find the sparse transform coefficients $\widehat{\mathbf{x}}$ and, then, reconstructing the signal $\mathbf{f}$ by a synthesis operation, that is, $\widehat{\mathbf{f}}=D \widehat{\mathbf{x}}$. This 
is the so-called $l_{1}$-synthesis or synthesis based method. Since the entries of $\Phi D$ are correlated when $D$ is highly coherent, $\Phi D$ may no longer satisfy the standard restricted isometry property (RIP [1]) and the mutual incoherence property (MIP [17]) which are commonly used in the standard CS framework. Thus, it would be difficult to characterize the theoretical performance of $l_{1}$-synthesis method under the CS framework.

An alternative to the $l_{1}$-synthesis method is the $l_{1}$-analysis method, which finds the estimator $\widehat{\mathbf{f}}$ directly by solving the following $l_{1}$ minimization problem:

$$
\begin{aligned}
\min _{\mathbf{f} \in \mathbb{R}^{n}} & \left\|\mathbf{D}^{*} \mathbf{f}\right\|_{1} \\
\text { subject to } & \|\Phi \mathbf{f}-\mathbf{y}\|_{2} \in B .
\end{aligned}
$$

It has been shown in [14] that there is a remarkable difference between the two typical methods despite their apparent similarity. To investigate the theoretical performance of the $l_{1}$-analysis method, Candès et al. in [13] introduced the definition of D-RIP: A measurement matrix $\Phi$ is said to satisfy the restricted isometry property adapted to $D$ (abbreviated $D$-RIP) with constant $\delta_{k}$ if

$$
\left(1-\delta_{k}\right)\|D \mathbf{x}\|_{2}^{2} \leq\|\Phi D \mathbf{x}\|_{2}^{2} \leq\left(1+\delta_{k}\right)\|D \mathbf{x}\|_{2}^{2}
$$

holds for every vector $\mathbf{x} \in \mathbb{R}^{d}$ that is $k$-sparse. Note that it is a natural generalization of the RIP introduced in [1]. Similarly, it is also computationally difficult to verify the $D$ RIP for a given deterministic matrix. But as discussed in [13], the matrices which satisfy the standard RIP requirements will also satisfy the $D$-RIP requirements. Many previous works have tried to derive sufficient conditions on $\delta_{c k}(c>0)$ for stable recovery of nearly sparse (in terms of $D$ ) signals via $l_{1}$ analysis. Candès et al. first presented conditions $\delta_{2 k}<0.08$ and $\delta_{7 k}<0.6$ [13]. Then, the conditions $9 \delta_{2 k}+4 \delta_{4 k}<5$ and $\delta_{2 k}<0.4931$ were used in [18] and [19], respectively. In the recent literature, S. Lin and J. Lin [19] extended the notion of restricted orthogonality constant (ROC) used in standard CS to the setting of CS with coherent tight frames. The $D$ restricted orthogonality constant (D-ROC) of order $k_{1}, k_{2}$, $\theta_{k_{1}, k_{2}}$ is defined to be the smallest positive number satisfying

$$
\left|\left\langle\Phi D \mathbf{x}_{1}, \Phi D \mathbf{x}_{2}\right\rangle-\left\langle D \mathbf{x}_{1}, D \mathbf{x}_{2}\right\rangle\right| \leq \theta_{k_{1}, k_{2}}\left\|\mathbf{x}_{1}\right\|_{2}\left\|\mathbf{x}_{2}\right\|_{2}
$$

for every $\mathbf{x}_{1}$ and $\mathbf{x}_{2}$ such that $\mathbf{x}_{1}$ and $\mathbf{x}_{2}$ are $k_{1}$-sparse and $k_{2}$-sparse, respectively. With this new notion, they extended some sufficient conditions which appeared in standard CS to the setting of CS with coherent tight frames, such as $\delta_{k}+$ $1.25 \theta_{k, k}<1, \delta_{(8 / 7) k}+\theta_{(8 / 7) k,(8 / 7) k}<1$, and $\delta_{1.25 k}+\theta_{k, 1.25 k}<1$. Moreover, they also obtained that $\delta_{k}<0.307$, which is the first sufficient condition on $\delta_{k}$, is sufficient for $l_{1}$-analysis to guarantee the stable recovery of nearly $k$-sparse (in terms of D) signals. In a recent paper [20], the condition $\delta_{k}<0.307$ was improved to $\delta_{k}<1 / 3$.

Along the lines of $[8,19]$, we establish in this paper more relaxed RIP conditions for stable recovery of nearly sparse (in terms of $D$ ) signals from incomplete and contaminated data. Specifically, the main contribution of this paper is to show that, under the RIP condition $\delta_{k}+\theta_{k, k}<1$, any signal that is nearly sparse in terms of $D$ can be recovered stably from its noisy measurements by solving the $l_{1}$-analysis problem (3). To show these new conditions, in Section 2, we shall introduce a key technique tool which is an extension of Lemma 5.1 in [8] and also state two lemmas that appeared in [8]. In Section 3, we will establish our new results by use of some proof ideas for the standard CS in [8]. Furthermore, we will show that the condition $\delta_{k}+\theta_{k, k}<1$ is mostly weaker than the best known condition $\delta_{k}<1 / 3$.

\section{Preliminaries}

In this section, we first state two useful lemmas that appeared in [8], which reveal the relationship between D-RIC and $D$-ROC, and the relationship between $D$-ROCs of different orders, respectively.

Lemma 1. For positive integers $k_{1}, k_{2} \leq d$, we have

$$
\theta_{k_{1}, k_{2}} \leq \delta_{k_{1}+k_{2}} \text {. }
$$

Lemma 2. For any $b \geq 1$ and positive integers $k_{1}, k_{2}$ such that $b k_{2}$ is an integer, we have

$$
\theta_{k_{1}, b k_{2}} \leq \sqrt{b} \theta_{k_{1}, k_{2}} .
$$

In the following, we will introduce and prove a key technical tool, which will be very useful for proving our main results.

Lemma 3. Let $k_{1}$ and $k_{2}$ and $\eta \geq 0$. Suppose $\mathbf{c}_{1}, \mathbf{c}_{2} \in \mathbb{R}^{d}$ and $\mathbf{c}_{1}$ is a $k_{1}$-sparse vector. If $\left\|\mathbf{c}_{2}\right\|_{1} \leq \eta k_{2}$ and $\left\|\mathbf{c}_{2}\right\|_{\infty} \leq \eta$, then we have

$$
\left|\left\langle\Phi D \mathbf{c}_{1}, \Phi D \mathbf{c}_{2}\right\rangle-\left\langle D \mathbf{c}_{1}, D \mathbf{c}_{2}\right\rangle\right| \leq \theta_{k_{1}, k_{2}}\left\|\mathbf{c}_{1}\right\|_{2} \cdot \eta \sqrt{k_{2}} .
$$

Proof. We shall prove it by mathematical induction. Suppose the size of support of $\mathrm{c}_{2}$ is $l$, that is, $\left\|\mathrm{c}_{2}\right\|_{0}=l$. For $l \leq k_{2}$, by the definition of $\theta_{k_{1}, k_{2}}$, we have

$$
\begin{gathered}
\left|\left\langle\Phi D \mathbf{c}_{1}, \Phi D \mathbf{c}_{2}\right\rangle-\left\langle D \mathbf{c}_{1}, D \mathbf{c}_{2}\right\rangle\right| \leq \theta_{k_{1}, k_{2}}\left\|\mathbf{c}_{1}\right\|_{2}\left\|\mathbf{c}_{2}\right\|_{2} \\
\leq \theta_{k_{1}, k_{2}}\left\|\mathbf{c}_{1}\right\|_{2} \sqrt{l\left\|\mathbf{c}_{2}\right\|_{\infty}^{2}} \leq \theta_{k_{1}, k_{2}}\left\|\mathbf{c}_{1}\right\|_{2} \cdot \eta \sqrt{k_{2}} .
\end{gathered}
$$

Thus, (8) holds for $l \leq k_{2}$.

For the case $l>k_{2}$, we first assume that (8) holds for $l-1$. The following discussion will use the same argument as in [8]. But for completeness, we will include the sketch. Now for $l$, we write $\mathbf{c}_{2}$ as $\mathbf{c}_{2}=\sum_{i=1}^{l} \alpha_{i} \Delta_{i}$, where $\alpha_{1} \geq \alpha_{2} \geq \cdots \geq \alpha_{l}>0$, and $\left\{\Delta_{i}\right\}_{i=1}^{l}$ are "indicator vectors" with different supports. A vector is called an "indicator vector" if it has only one nonzero entry and the value is either 1 or -1 . Since $\sum_{i=1}^{l} \alpha_{i} \leq \eta k_{2} \leq$ $(l-1) \eta$, the set

$$
\Omega \triangleq\left\{1 \leq j \leq l-1: \alpha_{j}+\alpha_{j+1}+\cdots+\alpha_{l} \leq(l-j) \eta\right\}
$$

is not empty. Now we choose the largest element $j \in \Omega$, which means

$$
\begin{gathered}
\alpha_{j}+\alpha_{j+1}+\cdots+\alpha_{l} \leq(l-j) \eta, \\
\alpha_{j+1}+\alpha_{j+2}+\cdots+\alpha_{l}>(l-j-1) \eta .
\end{gathered}
$$


Define

$$
\begin{aligned}
& \beta_{\omega}=\frac{\sum_{i=j}^{l} \alpha_{i}}{l-j}-\alpha_{\omega}, \quad j \leq \omega \leq l, \\
& \mathbf{r}_{\omega}=\frac{\beta_{\omega}}{\sum_{i=j}^{l} \beta_{i}} \sum_{i=1}^{j-1} \alpha_{i} \Delta_{i}+\sum_{i=j, i \neq \omega}^{l} \alpha_{i} \Delta_{i}, \quad j \leq i \leq l .
\end{aligned}
$$

It is not hard to check that $\sum_{\omega=j}^{l} \mathbf{r}_{\omega}=\mathbf{c}_{2}, \sum_{i=j}^{l}=(l-j) \sum_{i=j}^{l} \beta_{i}$. Similar to the proof of lemma 5.1 in [8], we also have

$$
\begin{aligned}
\beta_{\omega} & \geq \beta_{j}>0, \quad j \leq \omega \leq l, \\
\left\|\mathbf{r}_{\omega}\right\|_{1} & \leq \frac{\beta_{\omega}}{\sum_{i=j}^{l} \beta_{i}} \eta k_{2}, \\
\left\|\mathbf{r}_{\omega}\right\|_{\infty} & \leq \frac{\beta_{\omega}}{\sum_{i=j}^{l} \beta_{i}} \eta .
\end{aligned}
$$

From the definition of $\mathbf{r}_{\omega}$, we obtain that $\mathbf{r}_{\omega}$ is $(l-1)$-sparse. Finally, using the induction assumption, we get

$$
\begin{aligned}
& \left|\left\langle\Phi D \mathbf{c}_{1}, \Phi D \mathbf{c}_{2}\right\rangle-\left\langle D \mathbf{c}_{1}, D \mathbf{c}_{2}\right\rangle\right| \\
& \quad \leq \sum_{\omega=j}^{l}\left|\left\langle\Phi D \mathbf{c}_{1}, \Phi D \mathbf{r}_{\omega}\right\rangle-\left\langle D \mathbf{c}_{1}, D \mathbf{r}_{\omega}\right\rangle\right| \\
& \quad \leq \sum_{\omega=j}^{l} \theta_{k_{1}, k_{2}}\left\|\mathbf{c}_{1}\right\|_{2} \frac{\beta_{\omega}}{\sum_{i=j}^{l} \beta_{i}} \eta \sqrt{k_{2}} \\
& \quad=\theta_{k_{1}, k_{2}}\left\|\mathbf{c}_{1}\right\|_{2} \cdot \eta \sqrt{k_{2}},
\end{aligned}
$$

which arrives to the conclusion of Lemma 3.

Remark 4. When $D$ is an identity matrix and $\mathbf{c}_{1}, \mathbf{c}_{2}$ have disjoint supports, Lemma 3 has essentially the same result as lemma 5.1 in [8].

\section{Improved RIP Conditions}

We now consider the stable recovery of nearly sparse (in terms of $D$ ) signals via the $l_{1}$-analysis method (3). We will present some new RIP conditions under two bounded noise settings: $B_{1}(\varepsilon)=\left\{r:\|r\|_{2} \leq \varepsilon\right\}$ and $B_{2}(\varepsilon)=\left\{r:\left\|D^{*} \Phi^{*} r\right\|_{\infty} \leq\right.$ $\varepsilon\}$. Throughout the paper, $\mathbf{x}_{[k]}$ is the vector with all but the $k$ largest absolute entries of $\mathbf{x}$ set to zero, and $\mathbf{x}_{-[k]}=\mathbf{x}-\mathbf{x}_{[k]}$. The following theorem represents our main result.

Theorem 5. Let $D$ be a given tight frame, and $C_{a, b, k}=$ $\max \{(2 k-a) / \sqrt{a b}, \sqrt{(2 k-a) / a}\}$. If the measurement matrix $\Phi$ satisfies the D-RIP condition with

$$
\delta_{a}+C_{a, b, k} \theta_{a, b}<1
$$

for some positive integers $a$ and $b$ with $1 \leq a \leq k$, then the solution $\widehat{\mathbf{f}}$ to (3) obeys

(i) $\left(l_{2}\right.$ bounded noise)

$$
\begin{aligned}
\|\widehat{\mathbf{f}}-\mathbf{f}\|_{2} \leq & \frac{2 \varepsilon \sqrt{2\left(1+\delta_{a}\right) k / a}}{1-\delta_{a}-C_{a, b, k} \theta_{a, b}}+2\left\|D^{*} \mathbf{f}-\left(D^{*} \mathbf{f}\right)_{[k]}\right\|_{1} \\
& \times\left(\frac{\sqrt{2 k} C_{a, b, k} \theta_{a, b}}{\left(1-\delta-C_{a, b, k \theta_{a, b}}\right)(2 k-a)}+\frac{1}{\sqrt{k}}\right) .
\end{aligned}
$$

(ii) $\left(l_{\infty}\right.$ bounded noise)

$$
\begin{aligned}
\|\widehat{\mathbf{f}}-\mathbf{f}\|_{2} \leq & \frac{2 \varepsilon \sqrt{2 k}}{1-\delta_{a}-C_{a, b, k} \theta_{a, b}}+2\left\|D^{*} \mathbf{f}-\left(D^{*} \mathbf{f}\right)_{[k]}\right\|_{1} \\
& \times\left(\frac{\sqrt{2 k} C_{a, b, k} \theta_{a, b}}{\left(1-\delta-C_{a, b, k \theta_{a, b}}\right)(2 k-a)}+\frac{1}{\sqrt{k}}\right) .
\end{aligned}
$$

Remark 6. When $\mathbf{f}$ is exact $k$-sparse in terms of $D$ and no noise is present, the solution $\widehat{\mathbf{f}}$ to (3) is equal to $\mathbf{f}$; that is to say, the recovery is exact.

Remark 7. As reported in [8], when $D=I$, the bound 1 is sharp in the sense that, for any $\varepsilon>0$, the $D$-RIP condition $\delta_{a}+C_{a, b, k} \theta_{a, b}<1+\varepsilon$ does not guarantee such exact recovery. But it is still open whether this bound is also sharp when $D$ is not identity matrix. We leave it to the interested readers.

Proof. Let $\mathbf{h}=\widehat{\mathbf{f}}-\mathbf{f}$, where $\mathbf{f}$ is the original signal and $\widehat{\mathbf{f}}$ is the solution to (3). As noted in $[15,18]$, different from the proof in [8] for standard compressed sensing, we need to develop bounds on $\left\|D^{*} \mathbf{h}\right\|_{2}$ instead of $\|\mathbf{h}\|_{2}$. We write $D^{*} \mathbf{h}=$ $\sum_{i=1}^{d} \mu_{i} \Delta_{i}$, where $\mu_{1} \geq \mu_{2} \geq \cdots \geq \mu_{d} \geq 0$ and $\left\{\Delta_{i}\right\}_{i=1}^{d}$ are indicator vectors (we have mentioned in the proof of Lemma 3) with different support. In the following, we will use some proof ideas from [8].

By the fact that $\widehat{\mathbf{f}}$ is a minimizer of (3), we can easily get the following inequality (see $[5,6,19])$ :

$$
\sum_{i=k+1}^{d} \mu_{i} \leq \sum_{i=1}^{k} \mu_{i}+2\left\|\left(D^{*} \mathbf{f}\right)_{-[k]}\right\|_{1} .
$$

Thus,

$$
\begin{aligned}
\left\|\left(D^{*} \mathbf{h}\right)_{-[a]}\right\|_{\infty} & =\mu_{a+1} \leq \frac{\sum_{i=1}^{a} \mu_{i}}{a} \\
& \leq \frac{\sum_{i=1}^{a} \mu_{i}}{a}+\frac{2\left\|\left(D^{*} \mathbf{f}\right)_{-[k]}\right\|_{1}}{2 k-a}, \\
\left\|\left(D^{*} \mathbf{h}\right)_{-[a]}\right\|_{1} & =\sum_{i=a+1}^{k} \mu_{i}+\sum_{i=k+1}^{d} \mu_{i} \\
& \leq \sum_{i=a+1}^{k} \mu_{i}+\sum_{i=1}^{k} \mu_{i}+2\left\|\left(D^{*} \mathbf{h}\right)_{-[k]}\right\|_{1} .
\end{aligned}
$$


Note that

$$
\begin{aligned}
\sum_{i=a+1}^{k} \mu_{i} & \leq(k-a) \mu_{a+1} \leq \frac{k-a}{a} \sum_{i=1}^{a} \mu_{i} \\
\sum_{i=1}^{k} \mu_{i} & =\sum_{i=1}^{a} \mu_{i}+\sum_{i=a+1}^{k} \mu_{i} \leq \sum_{i=1}^{a} \mu_{i}+\frac{k-a}{a} \sum_{i=1}^{a} \mu_{i}=\frac{k}{a} \sum_{i=1}^{a} \mu_{i} .
\end{aligned}
$$

It then follows with (20) that

$$
\begin{aligned}
\left\|\left(D^{*} \mathbf{h}\right)_{-[a]}\right\|_{1} \leq & \frac{k-a}{a} \sum_{i=1}^{a} \mu_{i}+\frac{k}{a} \sum_{i=1}^{a} \mu_{i}+2\left\|\left(D^{*} \mathbf{h}\right)_{-[k]}\right\|_{1} \\
= & \frac{2 k-a}{a}\left\|\left(D^{*} \mathbf{h}\right)_{[a]}\right\|_{1} \\
& +2\left\|\left(D^{*} \mathbf{h}\right)_{-[k]}\right\|_{1} .
\end{aligned}
$$

Applying Lemma 3 with $\eta=\left\|\left(D^{*} \mathbf{h}\right)_{[a]}\right\|_{1} / a+2\left\|\left(D^{*} \mathbf{h}\right)_{-[k]}\right\|_{1} /$ $(2 k-a), k_{1}=a$ and $k_{2}=2 k-a$ yield

$$
\begin{aligned}
& \mid\left\langle\Phi D\left(D^{*} \mathbf{h}\right)_{[a]}, \Phi D\left(D^{*} \mathbf{h}\right)_{-[a]}\right\rangle \\
& \quad-\left\langle D\left(D^{*} \mathbf{h}\right)_{[a]}, D\left(D^{*} \mathbf{h}\right)_{-[a]}\right\rangle \mid \\
& \quad \leq \theta_{a, 2 k-a} \sqrt{2 k-a}\left\|\left(D^{*} \mathbf{h}\right)_{[a]}\right\|_{2} \times\left(\frac{\left\|\left(D^{*} \mathbf{h}\right)_{[a]}\right\|_{1}}{a}\right. \\
& \left.\quad+\frac{2\left\|\left(D^{*} \mathbf{h}\right)_{-[k]}\right\|_{1}}{2 k-a}\right) .
\end{aligned}
$$

Hence,

$$
\begin{aligned}
\langle\Phi \mathbf{h}, & \left.\Phi D\left(D^{*} \mathbf{h}\right)_{[a]}\right\rangle \\
= & \left\langle\Phi D\left(D^{*} \mathbf{h}\right)_{[a]}, \Phi D\left(D^{*} \mathbf{h}\right)_{[a]}\right\rangle \\
& +\left\langle\Phi D\left(D^{*} \mathbf{h}\right)_{-[a]}, \Phi D\left(D^{*} \mathbf{h}\right)_{[a]}\right\rangle \\
\geq & \left(1-\delta_{a}\right)\left\|D\left(D^{*} \mathbf{h}\right)_{[a]}\right\|_{2}^{2} \\
& -\theta_{a, 2 k-a} \sqrt{2 k-a}\left\|\left(D^{*} \mathbf{h}\right)_{[a]}\right\|_{2} \\
& \times\left(\frac{\left\|\left(D^{*} \mathbf{h}\right)_{[a]}\right\|}{a}+\frac{2\left\|\left(D^{*} \mathbf{h}\right)_{-[k]}\right\|_{1}}{2 k-a}\right) \\
& +\left\langle D\left(D^{*} \mathbf{h}\right)_{[a]}, D\left(D^{*} \mathbf{h}\right)_{-[a]}\right\rangle .
\end{aligned}
$$

By plugging the equality

$$
\begin{aligned}
& \left\langle D\left(D^{*} \mathbf{h}\right)_{[a]}, D\left(D^{*} \mathbf{h}\right)_{-[a]}\right\rangle \\
& =\left\langle D\left(D^{*} \mathbf{h}\right)_{[a]}, \mathbf{h}-D\left(D^{*} \mathbf{h}\right)_{[a]}\right\rangle \\
& =\left\langle D\left(D^{*} \mathbf{h}\right)_{[a]}, D D^{*} \mathbf{h}\right\rangle-\left\|D\left(D^{*} \mathbf{h}\right)_{[a]}\right\|_{2}^{2} \\
& =\left\|(D \mathbf{h})_{[a]}\right\|_{2}^{2}-\left\|D\left(D^{*} \mathbf{h}\right)_{[a]}\right\|_{2}^{2}
\end{aligned}
$$

into the above inequality, we obtain

$$
\begin{aligned}
\langle\Phi \mathbf{h}, & \left.\Phi D\left(D^{*} \mathbf{h}\right)_{[a]}\right\rangle \\
\geq & \left\|(D \mathbf{h})_{[a]}\right\|_{2}^{2}-\delta_{a}\left\|D\left(D^{*} \mathbf{h}\right)_{[a]}\right\|_{2}^{2} \\
& -\theta_{a, 2 k-a} \sqrt{2 k-a}\left\|\left(D^{*} \mathbf{h}\right)_{[a]}\right\|_{2} \\
& \times\left(\frac{\left\|\left(D^{*} \mathbf{h}\right)_{[a]}\right\|_{1}}{a}+\frac{2\left\|\left(D^{*} \mathbf{h}\right)_{-[k]}\right\|_{1}}{2 k-a}\right) \\
\geq & \left(1-\delta_{a}-\theta_{a, 2 k-a} \sqrt{\frac{2 k-a}{a}}\right)\left\|\left(D^{*} \mathbf{h}\right)_{[a]}\right\|_{2}^{2} \\
& -\theta_{a, 2 k-a}\left\|\left(D^{*} \mathbf{h}\right)_{[a]}\right\| \frac{2\left\|\left(D^{*} \mathbf{h}\right)_{-[k]}\right\|_{1}}{\sqrt{2 k-a}} .
\end{aligned}
$$

On the other hand, for $l_{2}$ bounded noise, we first have

$$
\begin{aligned}
\left\langle\Phi \mathbf{h}, \Phi D\left(D^{*} \mathbf{h}\right)_{[a]}\right\rangle & \leq\|\Phi \mathbf{h}\|_{2}\left\|\Phi D\left(D^{*} \mathbf{h}\right)_{[a]}\right\|_{2} \\
& \leq 2 \varepsilon \sqrt{1+\delta_{a}}\left\|D\left(D^{*} \mathbf{h}\right)_{[a]}\right\|_{2} \\
& \leq 2 \varepsilon \sqrt{1+\delta_{a}}\left\|\left(D^{*} \mathbf{h}\right)_{[a]}\right\|_{2},
\end{aligned}
$$

where we have used the following inequality:

$$
\|\Phi \mathbf{h}\|_{2} \leq\|\Phi \mathbf{h}-\mathbf{f}\|_{2}+\|\mathbf{f}-\Phi \mathbf{h}\|_{2} \leq 2 \varepsilon
$$

Therefore,

$$
\begin{aligned}
& \left\|\left(D^{*} \mathbf{h}\right)_{[a]}\right\|_{2} \\
& \leq \frac{2 \varepsilon \sqrt{1+\delta_{a}}}{1-\delta_{a}-\theta_{a, 2 k-a} \sqrt{(2 k-a) / a}} \\
& \quad+\frac{\theta_{a, 2 k-a}}{1-\delta_{a}-\theta_{a, 2 k-a} \sqrt{(2 k-a) / a}} \frac{2\left\|\left(D^{*} \mathbf{h}\right)_{-[k]}\right\|_{1}}{\sqrt{2 k-a}} .
\end{aligned}
$$

It is known that $\|\mathbf{h}\|_{2}^{2}=\left\|D^{*} \mathbf{h}\right\|_{2}^{2}$; thus, now we shall bound $\left\|D^{*} \mathbf{h}\right\|_{2}$. By lemma 5 in [7], we have

$$
\sum_{j=r+1}^{m} c_{j}^{2} \leq r\left(\sqrt{\frac{\sum_{i=1}^{r} c_{i}^{2}}{r}}+\frac{\lambda}{r}\right)^{2}
$$


if $c_{1} \geq c_{2} \geq \cdots \geq c_{m} \geq 0$, and $\sum_{i=1}^{r} c_{i}+\lambda \geq \sum_{i=r+1}^{m} c_{i}$ with $\lambda \geq 0$. Then, set $\lambda=2\left\|\left(D^{*} \mathbf{h}\right)_{-[k]}\right\|_{1}$ in (30) and combine it with (18); we further get

$$
\begin{aligned}
& \left\|D^{*} \mathbf{h}\right\|_{2}=\sqrt{\sum_{i=1}^{k} \mu_{i}^{2}+\sum_{i=k+1}^{d} \mu_{i}^{2}} \\
& \leq \sqrt{\sum_{i=1}^{k} \mu_{i}^{2}+k\left(\sqrt{\left.\sum_{i=1}^{k} \mu_{i}^{2}+\frac{2\left\|\left(D^{*} \mathbf{h}\right)_{-[k]}\right\|_{1}}{\sqrt{k}}\right)^{2}}\right.} \\
& \leq \sqrt{2 \sum_{i=1}^{k} \mu_{i}^{2}+\frac{2\left\|\left(D^{*} \mathbf{h}\right)_{-[k]}\right\|_{1}}{\sqrt{k}}} \\
& \leq \sqrt{\frac{2 k}{a} \sum_{i=1}^{a} \mu_{i}^{2}+\frac{2\left\|\left(D^{*} \mathbf{h}\right)\right\|_{1}}{\sqrt{k}}} \\
& \leq \frac{2 \varepsilon \sqrt{2\left(1+\delta_{a}\right) k / a}}{1-\delta_{a}-\theta_{k, 2 k-a} \sqrt{(2 k-a) / a}} \\
& +\left(\frac{\theta_{a, 2 k-a}}{1-\delta_{a}-\theta_{a, 2 k-a} \sqrt{(2 k-a) / a}} \frac{2}{\sqrt{2 k-a}}\right) \\
& \quad \cdot\left(D^{*} \mathbf{h}\right)_{-[k]},
\end{aligned}
$$

where the last inequality we have used is (29). From Lemma 2, it is not hard to get

$$
\begin{aligned}
\theta_{a, 2 k-a} & \leq \sqrt{\frac{2 k-a}{\min \{b, 2 k-a\}}} \theta_{a, \min \{b, 2 k-a\}} \\
& \leq \max \left\{\sqrt{\frac{2 k-a}{b}}, 1\right\} \theta_{a, b} \\
& =\sqrt{\frac{a}{2 k-a}} C_{a, b, k} \theta_{a, b} .
\end{aligned}
$$

Therefore,

$$
\begin{aligned}
\|\mathbf{h}\|_{2} \leq & \frac{2 \varepsilon \sqrt{2\left(1+\delta_{a}\right) k / a}}{1-\delta_{a}-C_{a, b, k} \theta_{a, b}}+2\left\|D^{*} \mathbf{f}-\left(D^{*} \mathbf{f}\right)_{[k]}\right\|_{1} \\
& \times\left(\frac{\sqrt{2 k} C_{a, b, k} \theta_{a, b}}{\left(1-\delta-C_{a, b, k \theta_{a, b}}\right)(2 k-a)}+\frac{1}{\sqrt{k}}\right),
\end{aligned}
$$

which arrives to the conclusion.

For $l_{\infty}$ bounded noise, we first have

$$
\begin{aligned}
\left\|D^{*} \Phi^{*} \Phi \mathbf{h}\right\|_{\infty} \leq & \left\|D^{*} \Phi^{*}(\mathbf{y}-\Phi \widehat{\mathbf{h}})\right\|_{\infty} \\
& +\left\|D^{*} \Phi^{*}(\Phi \mathbf{h}-\mathbf{y})\right\|_{\infty} \leq 2 \varepsilon .
\end{aligned}
$$

Hence,

$$
\begin{aligned}
\left\langle\Phi \mathbf{h}, \Phi D\left(D^{*} \mathbf{h}\right)_{[a]}\right\rangle & =\left\langle D^{*} \Phi^{*} \Phi \mathbf{h},(D \mathbf{h})_{[a]}\right\rangle \\
& \leq\left\|D^{*} \Phi^{*} \Phi \mathbf{h}\right\|_{\infty}\left\|(D \mathbf{h})_{[a]}\right\|_{1} \\
& \leq 2 \varepsilon \sqrt{a}\left\|(D \mathbf{h})_{[a]}\right\|_{2} .
\end{aligned}
$$

Then, the following proof is essentially the same, where we only need to replace (27) with (35).

To be noted, if we choose $a=b=k$ in Theorem 5 , we can naturally get the following result.

Corollary 8. If the measurement matrix satisfies $\delta_{k}+\theta_{k, k}<1$, then the solution $\widehat{\mathbf{f}}$ of (3) satisfies (16) and (17). In particular, if the original signal $\mathbf{f}$ is exact $k$-sparse in terms of $D$, the recovery is exact in the noiseless case.

Remark 9. It is obvious that the obtained condition $\delta_{k}+\theta_{k, k}<$ 1 is weaker than $\delta_{k}+1.25 \theta_{k, k}<1, \delta_{(8 / 7) k}+\theta_{(8 / 7) k,(8 / 7) k}<1$, and $\delta_{1.25 k}+\theta_{k, 1.25 k}<1$ which were used in [15].

Since most of the sufficient recovery conditions in the literature are based on $D$-RIC $\delta$ alone, it would be interesting to compare these conditions with $\delta_{k}+\theta_{k, k}<1$. For this, we shall present the following lemma which provides the $D$-ROC $\theta$ in terms of $D$-RIC $\delta$.

Lemma 10. Let $D$ be a given tight frame; then, $D-R O C \theta_{k, k}$ and D-RIC $\delta_{k}$ of the measurement matrix $\Phi$ satisfy

$$
\theta_{k, k} \leq \begin{cases}2 \delta_{k}, & \text { when } k \text { is even, } k \geq 2, \\ \frac{2 k}{\sqrt{k^{2}-1}} \delta_{k}, & \text { when } k \text { is odd, } k \geq 3 .\end{cases}
$$

Proof. The proof is similar to the proof of lemma 3.1 in [8]. For simplicity, we only present the proof sketch in the following. For two $k$-sparse (in terms of $D$ ) signals $\mathbf{f}_{\mathbf{1}}, \mathbf{f}_{\mathbf{2}} \in \mathbb{R}^{n}$, we can write $D \mathbf{f}_{1}$ and $D \mathbf{f}_{2}$ as

$$
\begin{aligned}
& D \mathbf{f}_{\mathbf{1}}=\sum_{i \in T_{1}} c_{i} e_{i}, \\
& D \mathbf{f}_{\mathbf{2}}=\sum_{i \in T_{2}} d_{i} e_{i},
\end{aligned}
$$

where $c_{i}>0, d_{i}>0, T_{1}$ is the support of $D^{*} \mathbf{f}_{1}, T_{2}$ is the support of $D^{*} \mathbf{f}_{2}$, and $e_{i}$ is the vector whose $i$ th entry equals \pm 1 and all the other ones equal zero.

Case 1 ( $k \geq 2$ is even). Without loss of generality, suppose $\mathbf{f}_{\mathbf{1}}$ and $\mathbf{f}_{\mathbf{2}}$ are normalized such that $\left\|\mathbf{D f _ { 1 }}\right\|_{2}=\left\|\mathbf{D f _ { 2 }}\right\|_{2}=1$. Divide $T_{1}$ and $T_{2}$ into two subsets such that $T_{1}=T_{11} \cup T_{12}, T_{2}=$ $T_{21} \cup T_{22}$, and $T_{11}, T_{12}, T_{21}, T_{22}$ are disjoint and $\left|T_{i j}\right| \leq k / 2$ for $i, j \in\{1,2\}$. Denote

$$
\begin{aligned}
\left(D \mathbf{f}_{\mathbf{1}}\right)_{i} & =\sum_{i \in T_{1 i}} c_{i} e_{i}, \\
D \mathbf{f}_{\mathbf{2}} & =\sum_{i \in T_{2 i}} d_{i} e_{i},
\end{aligned}
$$

$$
i=1,2 \text {. }
$$


Then, by the definition of $D$-RIP (4), we have

$$
\begin{aligned}
& \left\langle\Phi D \mathbf{f}_{\mathbf{1}}, \Phi D \mathbf{f}_{\mathbf{2}}\right\rangle=\sum_{i, j=1}^{2}\left\langle\Phi\left(D \mathbf{f}_{\mathbf{1}}\right)_{i}, \Phi\left(D \mathbf{f}_{\mathbf{2}}\right)_{j}\right\rangle=\frac{1}{4} \\
& \cdot \sum_{i, j=1}^{2} \mid\left\|\Phi\left(D \mathbf{f}_{\mathbf{1}}\right)_{i}+\Phi\left(D \mathbf{f}_{\mathbf{2}}\right)_{j}\right\|_{2}^{2} \\
& -\left\|\Phi\left(D \mathbf{f}_{\mathbf{1}}\right)_{i}-\Phi\left(D \mathbf{f}_{\mathbf{2}}\right)_{j}\right\|_{2}^{2} \mid \leq \frac{1}{4} \\
& \cdot \sum_{i, j=1}^{2}\left\{\left(1+\delta_{k}\right)\left\|\left(D \mathbf{f}_{\mathbf{1}}\right)_{i}+\left(D \mathbf{f}_{\mathbf{2}}\right)_{j}\right\|_{2}^{2}\right. \\
& \left.-\left(1-\delta_{k}\right)\left\|\left(D \mathbf{f}_{\mathbf{1}}\right)_{i}-\left(D \mathbf{f}_{\mathbf{2}}\right)_{j}\right\|_{2}^{2}\right\}=\frac{1}{4} \\
& +\sum_{i, j=1}^{2}\left\{4 \delta_{k}\left(\left\|\left(D \mathbf{f}_{\mathbf{1}}\right)_{i}\right\|_{2}^{2}+\left\|\left(D \mathbf{f}_{\mathbf{2}}\right)_{j}\right\|_{2}^{2}\right)\right. \\
& \left.+4\left\langle\left(D \mathbf{f}_{\mathbf{1}}\right)_{i},\left(D \mathbf{f}_{\mathbf{2}}\right)_{j}\right\rangle\right\}=\sum_{i, j=1}^{2} \delta_{k}\left(c_{i}^{2}+d_{i}^{2}\right) \\
& +\sum_{i, j=1}^{2}\left\langle\left(D \mathbf{f}_{\mathbf{1}}\right)_{i},\left(D \mathbf{f}_{\mathbf{2}}\right)_{j}\right\rangle=2 \delta_{k}+\left\langle D \mathbf{f}_{\mathbf{1}}, D \mathbf{f}_{\mathbf{2}}\right\rangle .
\end{aligned}
$$

Similarly, we have

$$
\left\langle\Phi D \mathbf{f}_{1}, \Phi D \mathbf{f}_{2}\right\rangle \geq-2 \delta_{k}+\left\langle D \mathbf{f}_{1}, D \mathbf{f}_{2}\right\rangle
$$

Then, from (39) and (40), we have

$$
\left|\left\langle\Phi D \mathbf{f}_{1}, \Phi D \mathbf{f}_{\mathbf{2}}\right\rangle-\left\langle D \mathbf{f}_{\mathbf{1}}, D \mathbf{f}_{\mathbf{2}}\right\rangle\right| \leq 2 \delta_{k}\left\|D \mathbf{f}_{\mathbf{1}}\right\|_{2}\left\|D \mathbf{f}_{2}\right\|_{2}
$$

Thus, $\theta_{k, k} \leq 2 \delta_{k}$.

Case 2 ( $k \geq 3$ is odd). Without loss of generality, suppose $\left|T_{1}\right|=k,\left|T_{2}\right|=k$, and $c_{i}, d_{i}$ might be 0 for $i \in T_{1} \cup$ $T_{2}$. Also we can assume $D \mathbf{f}_{1}$ and $D \mathbf{f}_{2}$ are normalized such that $\left\|\mathbf{D f}_{\mathbf{1}}\right\|_{2}=\sum_{i \in T_{1}} c_{i}^{2}=\sqrt{(k-1) /(k+1)}$ and $\left\|\mathbf{D f}_{\mathbf{2}}\right\|_{2}=$ $\sum_{i \in T_{2}} d_{i}^{2}=\sqrt{(k+1) /(k-1)}$. Then, we have

$$
\begin{aligned}
& 4\left(\begin{array}{c}
k-1 \\
\frac{(k-1)}{2}
\end{array}\right)\left(\begin{array}{c}
k-1 \\
\frac{(k-3)}{2}
\end{array}\right)\left\langle\Phi \mathbf{D f}_{1}, \Phi \mathbf{D f}_{2}\right\rangle \\
& =4\left(\begin{array}{c}
k-1 \\
\frac{(k-1)}{2}
\end{array}\right)\left(\begin{array}{c}
k-1 \\
\frac{(k-3)}{2}
\end{array}\right)\left\langle\Phi\left(\sum_{i \in T_{1}} c_{i} e_{i}\right),\right. \\
& \left.\Phi\left(\sum_{i \in T_{2}} d_{i} e_{i}\right)\right\rangle
\end{aligned}
$$

$$
\begin{aligned}
& =\sum_{\substack{A \subseteq T_{1},|A|=(k+1) / 2 \\
B \subseteq T_{2},\left|T_{2}\right|=(k-1) / 2}}\left\{\left\|\Phi\left(\sum_{i \in A} c_{i} e_{i}+\sum_{i \in B} d_{i} e_{i}\right)\right\|_{2}^{2}\right. \\
& \left.-\left\|\Phi\left(\sum_{i \in A} c_{i} e_{i}-\sum_{i \in B} d_{i} e_{i}\right)\right\|_{2}^{2}\right\} \\
& \leq \sum_{\substack{A \subseteq T_{1},|A|=(k+1) / 2 \\
B \subseteq T_{2},\left|T_{2}\right|=(k-1) / 2}}\left\{2 \delta_{k}\left(\sum_{i \in A} c_{i}^{2}+\sum_{i \in B} d_{i}^{2}\right)\right. \\
& \left.+4 \sum_{i \in A} \sum_{j \in B}\left\langle c_{i}, d_{j}\right\rangle\right\} \\
& =2 \delta_{k}\left\{\left(\begin{array}{c}
k-1 \\
\frac{(k-1)}{2}
\end{array}\right)\left(\begin{array}{c}
k \\
\frac{(k-1)}{2}
\end{array}\right) \sum_{i \in T_{1}} c_{i}^{2}\right. \\
& \left.+\left(\begin{array}{c}
k-1 \\
\frac{(k-3)}{2}
\end{array}\right)\left(\begin{array}{c}
k \\
\frac{(k+1)}{2}
\end{array}\right) \sum_{j \in T_{2}} d_{j}^{2}\right\} \\
& +4\left(\begin{array}{c}
k-1 \\
\frac{(k-1)}{2}
\end{array}\right)\left(\begin{array}{c}
k-1 \\
\frac{(k-3)}{2}
\end{array}\right) \sum_{i \in T_{1}} \sum_{j \in T_{2}}\left\langle c_{i}, d_{j}\right\rangle \\
& =2 \delta_{k}\left(\begin{array}{c}
k-1 \\
\frac{(k-1)}{2}
\end{array}\right)\left(\begin{array}{c}
k-1 \\
\frac{(k-3)}{2}
\end{array}\right)\left\{\frac{k}{(k-1) / 2} \sum_{i \in T_{1}} c_{i}^{2}\right. \\
& \left.+\frac{k}{(k+1) / 2} \sum_{j \in T_{2}} d_{j}^{2}\right\} \\
& +4\left(\begin{array}{c}
k-1 \\
\frac{(k-1)}{2}
\end{array}\right)\left(\begin{array}{c}
k-1 \\
\frac{(k-3)}{2}
\end{array}\right) \sum_{i \in T_{1}} \sum_{j \in T_{2}}\left\langle c_{i}, d_{j}\right\rangle \\
& =4\left(\begin{array}{c}
k-1 \\
\frac{(k-1)}{2}
\end{array}\right)\left(\begin{array}{c}
k-1 \\
\frac{(k-3)}{2}
\end{array}\right)\left\{\frac{2 k}{\sqrt{k^{2}-1}} \delta_{k}\left\|\mathbf{D f}_{\mathbf{1}}\right\|_{2}\right. \\
& \left.\cdot\left\|\mathrm{Df}_{2}\right\|_{2}+\left\langle\mathrm{Df}_{1}, \mathrm{Df}_{2}\right\rangle\right\} \text {. }
\end{aligned}
$$

Similarly, we have

$$
\begin{gathered}
4\left(\begin{array}{c}
k-1 \\
\frac{(k-1)}{2}
\end{array}\right)\left(\begin{array}{c}
k-1 \\
\frac{(k-3)}{2}
\end{array}\right)\left\langle\Phi D \mathbf{f}_{\mathbf{1}}, \Phi D \mathbf{f}_{\mathbf{2}}\right\rangle \\
\geq 4\left(\begin{array}{c}
k-1 \\
\frac{(k-1)}{2}
\end{array}\right)\left(\begin{array}{c}
k-1 \\
\frac{(k-3)}{2}
\end{array}\right)\left\{-\frac{2 k}{\sqrt{k^{2}-1}} \delta_{k}\left\|\mathbf{D f}_{\mathbf{1}}\right\|_{2}\right. \\
\left.\cdot\left\|\mathbf{D f}_{\mathbf{2}}\right\|_{2}+\left\langle\mathbf{D f}_{\mathbf{1}}, \mathbf{D f}_{\mathbf{2}}\right\rangle\right\} .
\end{gathered}
$$


Then, from (42) and (43), we have

$$
\begin{aligned}
& \left|\left\langle\Phi \mathbf{D f}_{\mathbf{1}}, \Phi \mathbf{D f}_{\mathbf{2}}\right\rangle-\left\langle\mathbf{D f}_{\mathbf{1}}, \mathbf{D f}_{\mathbf{2}}\right\rangle\right| \\
& \quad \leq \frac{2 k}{\sqrt{k^{2}-1}} \delta_{k}\left\|\mathbf{D f}_{\mathbf{1}}\right\|_{2}\left\|\mathbf{D f}_{\mathbf{2}}\right\|_{2},
\end{aligned}
$$

which implies $\theta_{k, k} \leq\left(2 k / \sqrt{k^{2}-1}\right) \delta_{k}$.

The following results can be directly obtained from the above Theorem 5 and Lemma 10.

Corollary 11. For some integer $k \geq 2$, if $\delta_{k}<1 / 3$, then we have

$$
\begin{aligned}
& \theta_{k, k}+\delta_{k}<1, \text { when } k \text { is even; } \\
& \theta_{k, k}+\delta_{k}<\frac{1}{3}+\frac{2 k}{3 \sqrt{k^{2}-1}}<1+\frac{1}{3 k^{2}},
\end{aligned}
$$

when $k$ is odd.

Remark 12. As stated in [20], $\delta_{k}<1 / 3$ is the best condition for stable recovery of nearly sparse signal in terms of $D$ via $l_{1}$ analysis. Based on Corollary 11, our presented condition $\theta_{k, k}+$ $\delta_{k}<1$ is mostly weaker than $\delta_{k}<1 / 3$. Basically, there are several benefits to weaken the $D$-RIP condition. For example, using a standard covering argument as in [21], it is easy to show that, for any positive integer $m<n$ and $0<t<1$, the $D$-RIC $\delta_{m}$ of a Gaussian or Bernoulli random measurement matrix satisfies

$$
P\left(\delta_{k}<t\right) \geq 1-2\left(\frac{12 e p}{m t}\right)^{m} \exp \left(-n\left(\frac{t^{2}}{16}-\frac{t^{3}}{48}\right)\right) .
$$

Note that $\theta_{k, k}+\delta_{k}<1$ is implied by $\delta_{2 k}+\delta_{k}<1$ which is further implied by the conditions $\delta_{k}<0.4$ and $\delta_{2 k}<0.6$. Hence, using (46) and following the discussion of Section IV in [8], the number of measurements $n$ should satisfy

$$
n \geq 162\left[k\left(\log \left(\frac{p}{k}\right)+4.6\right)-\log \left(\frac{\varepsilon}{2}\right)\right]
$$

to ensure the condition $\delta_{k}<1 / 3$ holds with probability at least $1-\varepsilon$. Similarly, $\delta_{k}+\delta_{2 k}<1$ holds with probability at least $1-\varepsilon$ if the number of measurements $n$ satisfies $n \geq \max \left\{n_{1}, n_{2}\right\}$ with

$$
n_{1}=115.4\left[k\left(\log \left(\frac{p}{k}\right)+4.4\right)-\log \left(\frac{\varepsilon}{4}\right)\right]
$$

guaranteeing $\delta_{k}<0.4$ with probability at least $1-\varepsilon / 2$, and with

$$
n_{2}=111.1\left[k\left(\log \left(\frac{p}{k}\right)+3.3\right)-\frac{\log (\varepsilon / 4)}{2}\right]
$$

guaranteeing $\delta_{2 k}<0.6$ with probability at least $1-\varepsilon / 2$. Therefore, for large $k$ and $p$, the size requirement to ensure $\theta_{k, k}+\delta_{k}<1$ is less than $71.2 \%(115.4 / 162)$ of the corresponding size requirement to ensure $\delta_{k}<1 / 3$. This clearly demonstrates the advantage of our presented condition $\theta_{k, k}+\delta_{k}<1$ over the best known condition $\delta_{k}<1 / 3$.

\section{Conclusion}

Under the framework of CS with coherent tight frames, we present in this paper some improved RIP conditions for stable recovery of nearly sparse (in terms of $D$ ) signals via $l_{1}$-analysis method, which are weaker than the existing ones. Although only convex optimization method is considered here, it would be also interesting to relax the RIP conditions for nonconvex optimization method. It is known that standard $l_{q}(0<$ $q<1)$ minimization method could recover conventional nearly sparse signals stably under weaker RIP conditions than standard $l_{1}$ minimization method [22-24]. As such, one may make effort to weaken the RIP condition for nonconvex $l_{q}(0<q<1)$-analysis method, thus facilitating the further use of nonconvex analysis based method for more practical CS scenarios.

\section{Conflicts of Interest}

The authors declare that there are no conflicts of interest regarding the publication of this paper.

\section{Acknowledgments}

This work was supported by Natural Science Foundation of China under Grant nos. 11501440, 61673015, and 62173020.

\section{References}

[1] E. J. Candès and T. Tao, "Decoding by linear programming," Institute of Electrical and Electronics Engineers. Transactions on Information Theory, vol. 51, no. 12, pp. 4203-4215, 2005.

[2] E. J. Candès, J. Romberg, and T. Tao, "Robust uncertainty principles: exact signal reconstruction from highly incomplete frequency information," Institute of Electrical and Electronics Engineers. Transactions on Information Theory, vol. 52, no. 2, pp. 489-509, 2006.

[3] D. L. Donoho, "Compressed sensing," Institute of Electrical and Electronics Engineers. Transactions on Information Theory, vol. 52, no. 4, pp. 1289-1306, 2006.

[4] E. J. Candes and T. Tao, "Near-optimal signal recovery from random projections: universal encoding strategies?" Institute of Electrical and Electronics Engineers. Transactions on Information Theory, vol. 52, no. 12, pp. 5406-5425, 2006.

[5] T. T. Cai, L. Wang, and G. Xu, "New bounds for restricted isometry constants," Institute of Electrical and Electronics Engineers. Transactions on Information Theory, vol. 56, no. 9, pp. 43884394, 2010

[6] T. T. Cai, G. Xu, and J. Zhang, "On recovery of sparse signals via $\ell_{1}$ minimization," Institute of Electrical and Electronics Engineers. Transactions on Information Theory, vol. 55, no. 7, pp. 3388-3397, 2009.

[7] T. T. Cai and A. Zhang, "Sharp RIP bound for sparse signal and low-rank matrix recovery," Applied and Computational Harmonic Analysis, vol. 35, no. 1, pp. 74-93, 2013.

[8] T. T. Cai and A. Zhang, "Compressed sensing and affine rank minimization under restricted isometry," IEEE Transactions on Signal Processing, vol. 61, no. 13, pp. 3279-3290, 2013. 
[9] M. Grasmair, "Non-convex sparse regularisation," Journal of Mathematical Analysis and Applications, vol. 365, no. 1, pp. 1928, 2010.

[10] M. Lustig, J. M. Santos, J. H. Lee, D. L. Donoho, and J. M. Pauly, "Application of compressed sensing for rapid MR imaging," in Proceedings of the SPARS, Rennes, France, 2005.

[11] B. K. Natarajan, "Sparse approximate solutions to linear systems," SIAM Journal on Computing, vol. 24, no. 2, pp. 227-234, 1995.

[12] H. Rauhut, K. Schnass, and P. Vandergheynst, "Compressed sensing and redundant dictionaries," Institute of Electrical and Electronics Engineers. Transactions on Information Theory, vol. 54, no. 5, pp. 2210-2219, 2008.

[13] E. J. Candès, Y. C. Eldar, D. Needell, and P. Randall, "Compressed sensing with coherent and redundant dictionaries," Applied and Computational Harmonic Analysis, vol. 31, no. 1, pp. 59-73, 2011.

[14] M. Elad, P. Milanfar, and R. Rubinstein, "Analysis versus synthesis in signal priors," Inverse Problems. An International Journal on the Theory and Practice of Inverse Problems, Inverse Methods and Computerized Inversion of Data, vol. 23, no. 3, pp. 947-968, 2007.

[15] J. Lin, S. Li, and Y. Shen, "New bounds for restricted isometry constants with coherent tight frames," IEEE Transactions on Signal Processing, vol. 61, no. 3, pp. 611-621, 2013.

[16] J. Lin and S. Li, "Sparse recovery with coherent tight frames via analysis Dantzig selector and analysis LASSO," Applied and Computational Harmonic Analysis. Time-Frequency and TimeScale Analysis, Wavelets, Numerical Algorithms, and Applications, vol. 37, no. 1, pp. 126-139, 2014.

[17] D. L. Donoho, M. Elad, and V. N. Temlyakov, "Stable recovery of sparse overcomplete representations in the presence of noise," Institute of Electrical and Electronics Engineers. Transactions on Information Theory, vol. 52, no. 1, pp. 6-18, 2006.

[18] Y. Liu, T. Mi, and S. Li, "Compressed sensing with general frames via optimal-dual-based $\ell_{1}$-analysis," Institute of Electrical and Electronics Engineers. Transactions on Information Theory, vol. 58, no. 7, pp. 4201-4214, 2012.

[19] S. Li and J. Lin, "Compressed sensing with coherent tight frames via $\ell_{q}$-minimization for $0<q \leq 1$," Inverse Problems and Imaging, vol. 8, no. 3, pp. 761-777, 2014.

[20] R. Zhang and S. Li, "Optimal D-RIP bounds in compressed sensing," Acta Mathematica Sinica (English Series), vol. 31, no. 5, pp. 755-766, 2015.

[21] R. Baraniuk, M. Davenport, R. DeVore, and M. Wakin, "A simple proof of the restricted isometry property for random matrices," Constructive Approximation. An International Journal for Approximations and Expansions, vol. 28, no. 3, pp. 253-263, 2008.

[22] R. Chartrand, "Exact reconstruction of sparse signals via nonconvex minimization," IEEE Signal Processing Letters, vol. 14, no. 10, pp. 707-710, 2007.

[23] Q. Sun, "Recovery of sparsest signals via $\ell_{q}$-minimization," Applied and Computational Harmonic Analysis. Time-Frequency and Time-Scale Analysis, Wavelets, Numerical Algorithms, and Applications, vol. 32, no. 3, pp. 329-341, 2012.

[24] C.-B. Song and S.-T. Xia, "Sparse signal recovery by $\ell_{q}$ minimization under restricted isometry property," IEEE Signal Processing Letters, vol. 21, no. 9, pp. 1154-1158, 2014. 


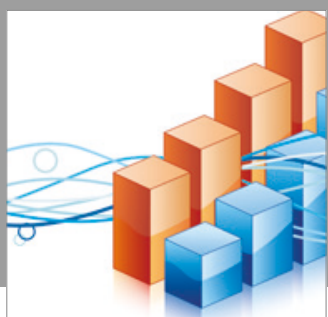

Advances in

Operations Research

vatersals

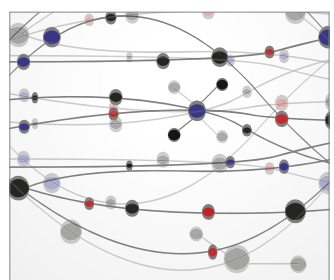

\section{The Scientific} World Journal
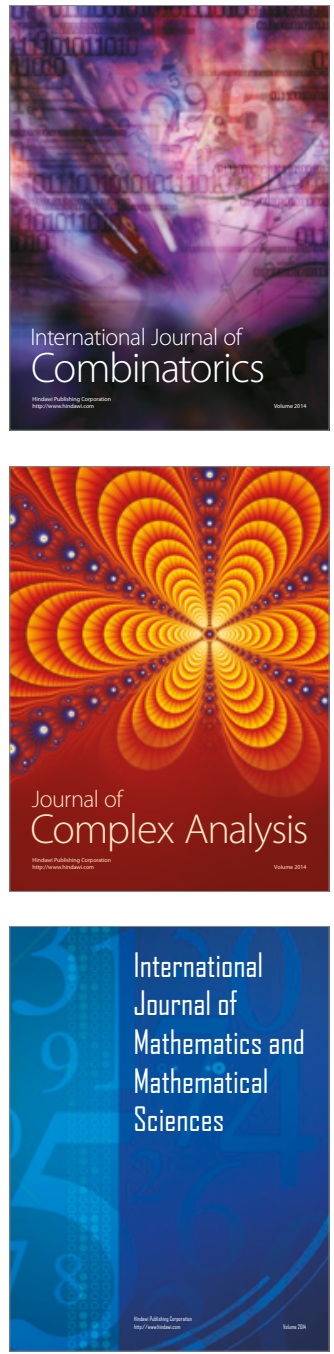
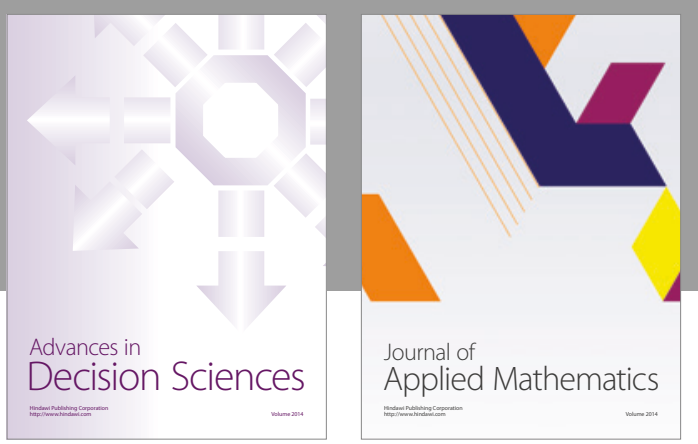

Algebra

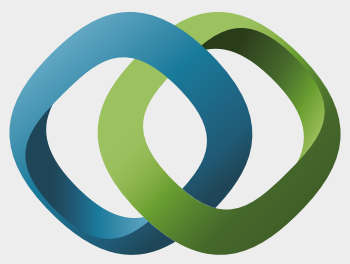

\section{Hindawi}

Submit your manuscripts at

https://www.hindawi.com
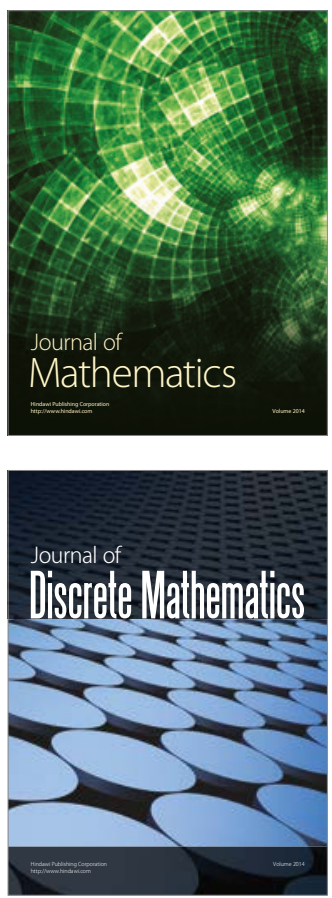

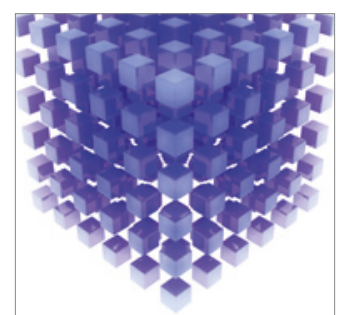

Mathematical Problems in Engineering
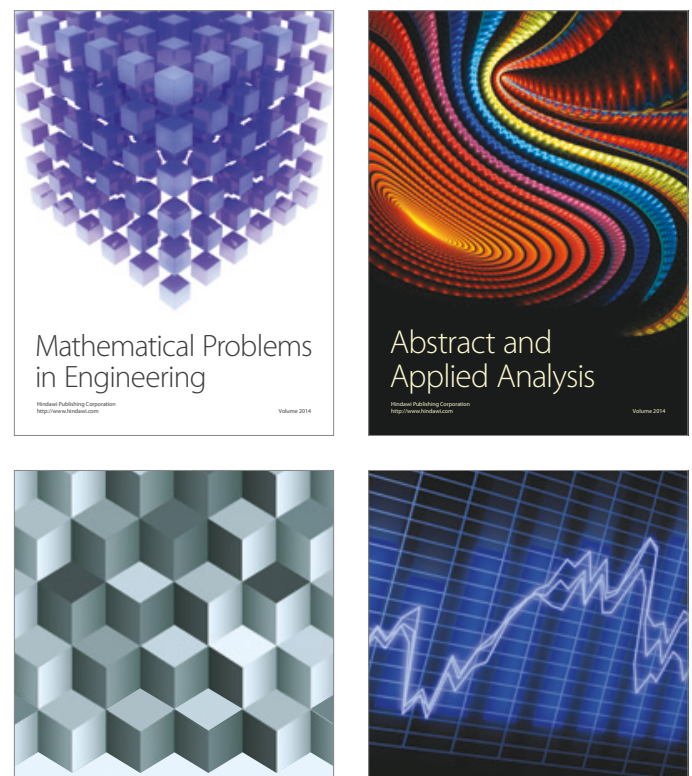

Journal of

Function Spaces

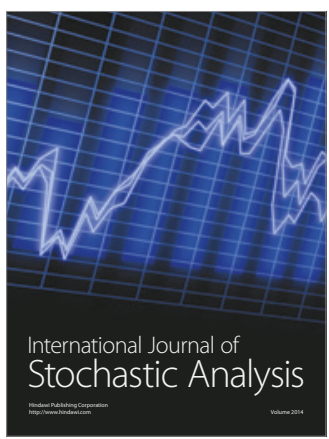

Probability and Statistics
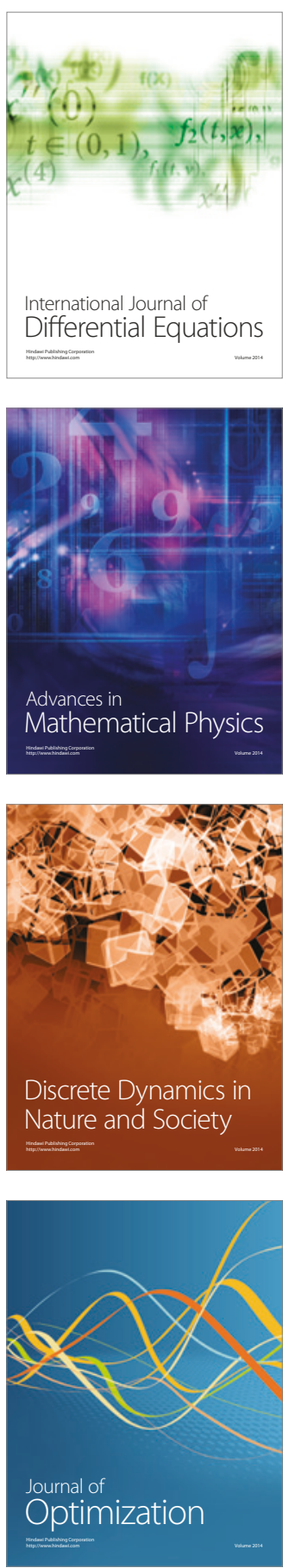